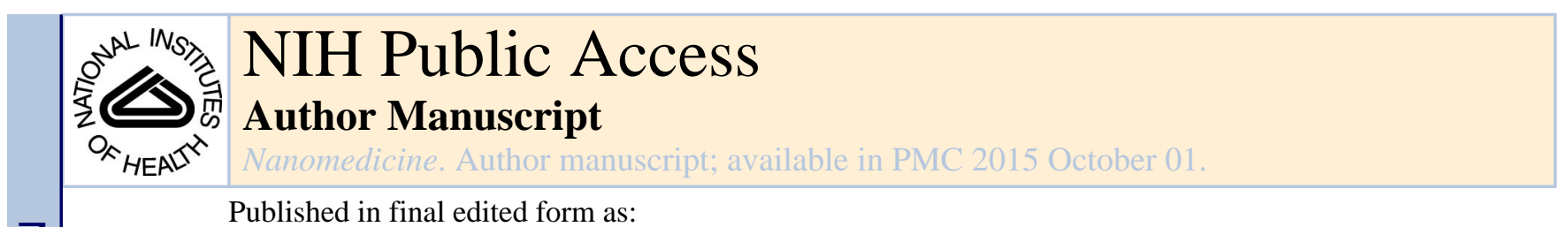

Published in final edited form as:

Nanomedicine. 2014 October ; 10(7): 1497-1506. doi:10.1016/j.nano.2014.03.016.

\title{
Development of a Novel Orthotopic Non-small Cell Lung Cancer Model and Therapeutic Benefit of 2'-(2-bromohexadecanoyl)- Docetaxel Conjugate Nanoparticles
}

\author{
Lei Peng, PhD ${ }^{a}$, Lan Feng, PhD $^{a}$, Hong Yuan, PhD ${ }^{b, c}$, Soumya R. Benhabbour, PhD ${ }^{a}$, and \\ Russell J. Mumper, PhDa,d, \\ aCenter for Nanotechnology in Drug Delivery, Division of Molecular Pharmaceutics, UNC \\ Eshelman School of Pharmacy, University of North Carolina at Chapel Hill, Chapel Hill, NC \\ 27599, USA \\ ${ }^{b}$ Department of Radiology, University of North Carolina at Chapel Hill, Chapel Hill, NC 27599, \\ USA
}

'Biomedical Research Imaging Center, University of North Carolina at Chapel Hill, Chapel Hill, NC 27599, USA

dUNC Lineberger Comprehensive Cancer Center, University of North Carolina at Chapel Hill, Chapel Hill, NC 27599, USA

\begin{abstract}
The aims of these studies were to establish an orthotopic non-small-cell lung cancer (NSCLC) mouse model, and to investigate the therapeutic efficacy of lipid-based nanoparticles (NPs) containing 2'-(2-bromohexadecanoyl)-docetaxel (Br-C16-DX) in this new model. A novel orthotopic NSCLC model was established in nude mice through dorsal side injection of luciferaseexpressing A549 cells. The model was characterized by survival study, histological staining, bioluminescence imaging and PET/CT imaging. The therapeutic efficacy of the Br-C16-DX NPs versus Taxotere ${ }^{\circledR}$ was investigated in this model. The results demonstrated that mouse survival time was significantly prolonged by weekly intravenous administration of the NPs or Taxotere. Furthermore, the NPs group had 35 days longer progression-free survival and 27 days longer median survival compared to the Taxotere group. It was concluded that the developed orthotopic NSCLC model represents a feasible, reproducible, and clinically-relevant experimental mouse model to test current and potential therapies including nanomedicines.
\end{abstract}

(C) 2014 Elsevier Inc. All rights reserved.

*Corresponding author: Russell J. Mumper, Ph.D., Vice Dean and John A. McNeill Distinguished Professor, Division of Molecular Pharmaceutics, UNC Eshelman School of Pharmacy, CB\# 7355, 100G Beard Hall, University of North Carolina at Chapel Hill, Chapel Hill, North Carolina 27599-7355, mumper@email.unc.edu, Phone: (919) 966-1271, Fax: (919) 966-6919.

The authors report no commercial association or conflict of interest for the research in this article.

Publisher's Disclaimer: This is a PDF file of an unedited manuscript that has been accepted for publication. As a service to our customers we are providing this early version of the manuscript. The manuscript will undergo copyediting, typesetting, and review of the resulting proof before it is published in its final citable form. Please note that during the production process errors may be discovered which could affect the content, and all legal disclaimers that apply to the journal pertain. 


\section{Keywords}

orthotopic lung cancer model; docetaxel conjugate; lipid nanoparticles; bioluminescence; micro $\mathrm{PET} / \mathrm{CT}$

\section{Background}

Lung cancer is the second most common cancer type and the leading cause of cancer mortality worldwide. The estimated death caused by lung cancer in 2012 is $29 \%$ for males and $26 \%$ for females in United States. ${ }^{1}$ Non-small cell lung cancer (NSCLC) including squamous cell carcinoma, adenocarcinoma, and large cell carcinoma accounts for $85 \%$ of all lung cancer cases.

The average five-year survival of NSCLC is only $15 \%$ with current therapies. ${ }^{1}$ Safer and more effective treatment options are in great demand. A major hurdle of lung cancer research for novel therapeutics has been lack of an easily feasible, reproducible, and clinically-relevant experimental mouse model. Orthotopic models have the advantage over ectopic models in that tumor grows in a microenvironment similar to clinical condition. Lack of metastasis and altered drug responses have been reported in commonly used subcutaneous tumors, which makes orthotopic models more favorable. ${ }^{2,3}$ Reported methods to establish orthotopic lung cancer mouse models with cell injection include injection through the trachea or chest wall. ${ }^{4-7}$ However, a majority of the studies are still performed in subcutaneous models due to lack of feasibility and reproducibility of previously reported models. ${ }^{8}$ In this paper, we report our findings wherein we established and characterized a bioluminescent orthotopic NSCLC model which provides high lung tumor development rate, low surgery mortality, good heart protection, and reliable bioluminescence signal for long term tumor growth monitoring.

Among the standard of care for NSCLC, docetaxel (DX) has been utilized either in combination with platinum-based drug or as a single agent. ${ }^{9}$ Similar to other lipophilic chemotherapy agents, its use has been limited by low water solubility, severe side-effects, and drug resistance. Side effects of DX such as anemia, allergy and low white blood cell count are partially caused by polysorbate 80 and ethanol in its commercialized form Taxotere $^{\circledR}$ (Sanofi-Aventis, Bridgewater NJ). Nanosystems including liposomes, micelles and nanoparticles have been investigated as safer formulations for DX delivery, where the use of polysorbate 80 and ethanol is avoided. Nanosystems provide advantages of decreased toxicity, prolonged circulating time, enhanced permeation and retention (EPR) effect, and potential targeted delivery. ${ }^{10-13}$ However, most DX formulations still face one or both major limitations of moderate drug solubility and rapid drug release in vivo.

Previously, our group investigated the possibility to deliver DX with our lipid-based BTM NPs (abbreviation from NP components Brij 78, Vitamin E TPGS and Miglyol 808).

Although DX has low water solubility, its solubility and affinity to the Miglyol oil core of BTM NPs was not high enough, leading to relatively low drug entrapment of DX and rapid drug release in vivo. To increase lipophilicity and subsequently drug entrapment and drug retention, a series of lipid-ester conjugates of DX with different fatty-acid chain lengths (12, 
18 , and 22) were successfully synthesized and incorporated into BTM NPs with significantly higher entrapment efficiencies (50-60\%). ${ }^{14}$ However, a common problem with taxane derivatives is the compromised cytotoxicity due to sub-optimal hydrolysis kinetics of these conjugates to release the parent drug. To overcome this limitation, a novel 2-Br-C16-DX conjugate was synthesized in more recent studies, where the presence of a bromine $(\mathrm{Br})$ at the 2-position of the fatty acid chain leads to faster hydrolysis kinetics to release DX. ${ }^{15}$ Therapeutic efficacy of this system was investigated in the developed orthotopic NSCLC model. The Br-C16-DX NPs had superior therapeutic efficacy over Taxotere, as evidenced by the delayed tumor-relapse and prolonged overall survival.

\section{Methods}

\section{Materials}

D-luciferin potassium salt, Caliper IVIS Lumina II and Living Image software were from Caliper (Hopkinton, Massachusetts). RPMI 1640, DPBS were purchased from Invitrogen (Carlsbad, California). Matrigel was purchased from BD Biosciences (San Jose, California). Primary antibodies for multidrug resistance-associated protein 1 (MRP1), lung resistancerelated protein (LRP) and p-glycoprotein (Pgp) were purchased from BIOSS (Woburn, Massachusetts). Primary antibody for glutathione S-transferase $\pi$ (GST- $\pi$ ) was purchased from Millipore (Billerica, Massachusetts). Polyoxyl 20-stearyl ether (Brij 78) was purchased from Uniqema (Wilmington, Delaware). D-alpha-tocopheryl polyethylene glycol-1000 succinate (Vitamin E-TPGS) was purchased from Eastman Chemicals (Kingsport, Tennessee). Miglyol 808 was purchased from Sasol (Witten, Germany). Docetaxel Injection Concentrate was purchased from Winthrop (Bridgewater, New Jersey).

\section{Cell culture}

Luciferase-expressing human lung adenocarcinoma cell line A549-luc-c8 (Caliper, Hopkinton, Massachusetts) was maintained in RPMI 1640 supplemented with 10\% FBS. Cells were cultured in a humid incubator with $5 \% \mathrm{CO}_{2}$ at $37^{\circ} \mathrm{C}$.

\section{Orthotopic lung tumor implantation and survival study}

Four to six week old female nude mice (Harlan Sprague Dawley) were housed in a pathogen-free room. Mice were anesthetized with $100 \mathrm{mg} / \mathrm{kg}$ ketamine i.p., $1 \mathrm{mg} / \mathrm{kg}$ domitor i.p., and topical $0.33 \%$ lidocaine hydrochloride. A $5 \mathrm{~mm}$ incision was made on dorsal side over left lung, about $1.5 \mathrm{~cm}$ above the lower rib line. Fat and muscles were separated to visualize lung movement. Luciferase expressing A549-luc-c8 cells suspended in 30 to $40 \mu \mathrm{l}$ $\mathrm{PBS} /$ matrigel were injected directly into left lung parenchyma at the depth of $3 \mathrm{~mm}$. The wound was closed with a surgery clip, which was removed 7 days later. After tumor cell injection, $2.5 \mathrm{mg} / \mathrm{kg}$ Antisedan was administered subcutaneously for the reversal from sedation. Mice were placed on a heating pad during and after the surgery procedure until they recover from anesthesia. One million A549-luc-c8 cells were injected per mouse during initial method development. However, in survival studies $(\mathrm{n}=34)$, five million A549-luc-c8 cells were injected per mouse to achieve desirable survival time. All mouse studies were conducted according to a protocol approved by the University of North Carolina 
Institutional Animal Care and Use Committee. Mice were sacrificed when Body Condition Scoring was 2 or less, or at $20 \%$ weight loss. ${ }^{16}$

\section{Whole-body bioluminescence imaging}

Weekly whole-body bioluminescence imaging was performed in mouse studies to monitor orthotopic lung tumor growth. D-luciferin potassium salt reconstituted in DPBS was injected intraperitoneally at $150 \mathrm{mg} / \mathrm{kg}$. Mice were anesthetized with $2 \%$ isoflurane and imaged by Caliper IVIS Lumina II fifteen minutes after luciferin injection. The Region of Interest (ROI) was defined as $3.2 \mathrm{~cm}$ radius circle over lung area. Total flux (photos/s) and average radiance $\left(\mathrm{p} / \mathrm{s} / \mathrm{cm}^{2} / \mathrm{sr}\right)$ within ROI were quantified using Living Image.

\section{Micro PET/CT imaging and correlation with bioluminescence imaging}

Female nude mice were implanted with five million A549-luc-c8 cells per mouse as described above $(n=4)$. Tumor-bearing mice were first imaged in the in vivo bioluminescence imaging system (IVIS-Kinetic, Perkin-Elmer, Inc.) using the protocol described earlier. MicroPET imaging was then performed on the same day on those animals using a small animal PET/CT scanner (eXplore Vista, GE Healthcare, Inc., Waukesha, Wisconsin) with a center resolution of $1.2 \mathrm{~mm}$. Animals were anesthetized by isofluorane mixed with oxygen. [18]F-Fluorodeoxyglucose (FDG) with an average dose of $7.2 \mathrm{MBq}$ was injected through the tail vein catheter. A CT scan was first acquired for anatomical localization and subsequent attenuation correction. PET acquisition was started at $30 \mathrm{~min}$ after FDG injection and continued for $10 \mathrm{~min}$. The optical and PET imaging was repeated on the same animal every week for at least four weeks. Raw PET data were reconstructed using 2D ordered subset expectation maximization (OSEM) algorithms with scatter, random, and attenuation correction using the manufacturer proprietary software. A standardized uptake value (SUV) was calculated based on the injection dose and animal body weight. ROI was manually drawn on the registered CT images along the rib cage to include the majority of the lung but exclude heart tissue. Mean SUVs of the lung ROI at different time points were reported, and compared to total flux (photos/s) of bioluminescence from the same mice.

\section{Histology staining}

At the time of sacrifice, various organs (lungs, heart, liver, spleen, kidney, brain) from the mice were harvested and fixed in 10\% formalin. After paraffin embedding, Hematoxylin \& Eosin (H\&E) staining, Masson's Trichrome staining, or immunohistochemistry staining (MRP1, LRP, Pgp and GST- $\pi$ ) were performed for $4 \mu \mathrm{m}$ sections by the Animal Histopathology Core at UNC-Chapel Hill. Microscopic observations were carried out with Olympus Bx-61 in Microscopy Services Laboratory at UNC-Chapel Hill.

\section{Preparation and characterization of BTM NPs with Br-C16-DX}

As previously reported, Br-C16-DX was synthesized from DX (Sigma-Aldrich, St. Louis, Missouri) to enhance lipophilicity, drug entrapment and drug retention in BTM NPs. ${ }^{17,} 18$ Briefly, DX was conjugated with 2-bromohexadecanoic acid (Sigma-Aldrich, St. Louis, Missouri) via a one-step esterification reaction. The product was purified by preparative TLC, and confirmed by TLC, NMR, and mass spectrometry. ${ }^{18}$ 
NPs with Br-C16-DX were prepared from a warm oil-in-water microemulsion precursor developed and reported previously with Brij 78, Vitamin E TPGS and Miglyol 808, and abbreviated as BTM NPs. ${ }^{19-21}$ Particle diameter and zeta potential of the NPs were characterized as previously reported. ${ }^{14}$ Drug entrapment efficiency was determined by size exclusion chromatography with Sepharose CL-4B, and the Br-C16-DX concentration was quantified by HPLC. ${ }^{18}$

\section{Efficacy study with Taxotere and Br-C16-DX NPs}

Five million A549-luc-c8 cells were implanted by dorsal side injection on day 0 as described above. Taxotere at $16 \mathrm{mg} / \mathrm{kg}$ or the Br-C16-DX NPs at $56 \mathrm{mg} / \mathrm{kg}$ of the conjugate (established maximum tolerated dose, MTD) were injected through the tail vein starting on day 5. Six weekly injections were performed on day 5, 12, 19, 26, 33 and 40. Weekly wholebody bioluminescence imaging was performed from day 0 through the end of the study. After mice were sacrificed, tissues were fixed in formalin for histological examination as described above.

\section{Statistical analysis}

Student's t-test was performed for two-group comparison, and Log-rank Test was performed for survival comparison (Prism, Version 5.01, GraphPad Software Inc., La Jolla, CA, USA). Differences were considered statistically significant when $\mathrm{p}<0.05$.

\section{Results}

\section{Orthotopic lung tumor implantation and survival study}

Luciferase-expressing A549-luc-c8 cells were used to establish orthotopic lung tumor model since luminescence signal allowed for injection verification as well as semi-quantification of tumor size and growth pattern. In vitro characterization proved a linear relationship between quantified luminescence level and cell number (supplementary Figure $S 1, R^{2}$ 0.9976), which provided a basis for utilizing bioluminescence level to monitor in vivo tumor growth.

The tumor implantation procedure is shown in Figure 1A. After a mouse was anesthetized, a $5 \mathrm{~mm}$ incision was made on dorsal side over left lung. A549-luc-c8 cells suspended in matrigel/PBS were injected into the left lung parenchyma to a depth around $3 \mathrm{~mm}$ and the wound was closed with a skin clip. A survival study with 34 nude mice was carried out to validate this tumor implantation method on a large animal set. With the dorsal side injection technique, surgery mortality was $0 \%$ and lung tumor development rate was as high as $94 \%$.

In studies investigating drug delivery system for cancer therapy, reasonable survival time in the mouse model needs to be tuned and validated. In a study by Madero-Visbal et al., it was reported that the median survival with one million A549 cells per mouse was around 60 days. ${ }^{22}$ In these current studies, we increased the number of cells injected to five million cells per mouse, which resulted in a median survival of 32 days (Figure 1B). Quantified in vivo bioluminescence increased in the beginning as tumor cells grew, then plateaued, followed by a continuous increase until sacrifice. The "increase - decrease or plateau continuous increase" pattern of bioluminescence level was observed repeatedly in all studies 
performed with this model. Lungs were harvested and gross images are shown in Figure 1C. Unlike a healthy lung with smooth surface, tumor-bearing lungs showed the presence of apparent disorganized tumor tissues. Pleural effusion was often observed in tumor-bearing lungs at the time of sacrifice.

\section{Histology characterization of the A549-luc-c8 tumor}

H\&E staining (Figure 2A-D) and Masson's Trichrome staining (Figure 2E-H) were performed in formalin-fixed tissues to further confirm tumor growth in the lung. Healthy lungs were well organized with single-cell-layer alveoli, which provided large surface area for air exchange and normal lung function. In contrast, tumor-bearing lungs presented disrupted alveolar structure, and tumor cells with large, pale nuclei. Immune cell infiltration and excess fibrin production in extracellular matrix were also observed in tumor-bearing lungs, which further compromised lung function. In Masson's Trichrome staining, a large amount of blue collagen was seen in tumor-bearing lungs but not healthy lungs, reflecting fibrosis during tumor growth.

The orthotopic lung tumor model was characterized for drug resistance due to its importance in responses to chemotherapy. Several resistance-related proteins including MRP1, Pgp, LRP and GST- $\pi$ were stained by immunohistochemistry. MRP1 and Pgp are efflux transporters of the ATP-binding cassette protein superfamily, which utilize energy from ATP hydrolysis to transport toxins or drugs out of the cell. LRP is a membrane transporter not belonging to the ATP-binding cassette superfamily. GST- $\pi$ is a transferase involved in drug detoxification pathway. As shown in Figure 2I-L, expressions of GST- $\pi$, MRP1 and LRP, but not Pgp, were observed.

\section{Micro PET/CT imaging}

Micro PET/CT imaging was performed in addition to 2D optical bioluminescence imaging (BLI) to obtain accurate 3D quantification in terms of tumor formation and metabolic activity. As shown in Figure 3 upper panel, abnormal neoplasma in the lung was shown by CT since day 21. Increasing FDG uptake signal in the lung was observed overtime (middle panel), indicating higher glucose metabolic activities due to tumor growth. Lymph nodes had high FDG uptake on day 41, implying metastasis of tumor cells. Weekly optical and PET imaging was performed for more than four weeks and the quantified signals showed increasing tumor burden overtime (Figure 3, lower panel). The relatively comparable signal from day 7 to day 14 in BLI is likely attributable to limited tissue penetration of bioluminescence signal and possible formation of a necrotic center in the tumor. Correlation between FDG SUV in the lung from PET images and the bioluminescence photon level in the lung from the same mouse was assessed (Figure 3, lower panel). A strong linear correlation was observed for quantified SUV and bioluminescence levels from four different mice over more than four weeks $\left(\mathrm{R}^{2}=0.86\right)$. This correlation not only helped to validate the orthotopic model but also supported the use of BLI to monitor tumor growth and therapeutic response over time in the efficacy studies. 


\section{Preparation and chracterization of BTM NPs containing Br-C16-DX}

Br-C16-DX (Figure 4) was synthesized as previously described and formulated in BTM NPs. ${ }^{18}$ The long 16-carbon chain increases the solubility of the conjugate in the oil core of the NPs, and the bromine $(\mathrm{Br})$ atom at the 2-position of the fatty acid chain leads to faster hydrolysis kinetics to release docetaxel. The Br-C16-DX NPs had a drug entrapment of 56.8 $\pm 2.8 \%$, mean particle diameter of $210 \pm 2.15 \mathrm{~nm}$, and zeta potential of $-14.0 \pm 0.9 \mathrm{mV}$ in 0.05 molar phosphate buffer. ${ }^{18}$ In vitro release studies of Br-C16-DX NPs were performed in $100 \%$ mouse plasma. An initial 40-45\% burst release of Br-C16-DX was observed within 1 hour with little or no additional release over the next 8 hours. ${ }^{18}$

\section{In vivo anti-tumor efficacy}

The therapeutic efficacy of Br-C16-DX NPs was evaluated in the A549-luc-c8 orthotopic lung tumor mouse model. Taxotere (16 mg/kg) or Br-C16-DX NPs $(56 \mathrm{mg} / \mathrm{kg}$ of Br-C16DX) was administered intravenously at predetermined MTD starting day 5 post-tumor implantation. The control group was left untreated ( $n=9$ in each group). Similar to the survival study, the average bioluminescence in the control group increased in the beginning, and then decreased, followed by a continuous increase (Figure 5A). BLI analysis showed significantly lower luminescence levels in the Br-C16-DX NPs treatment group compared to the control untreated group starting at day 13. The luminescence intensity in the Taxotere group reached significantly lower levels compared to the untreated group one week later, at day 20. Furthermore, the average luminescence level on day 20 in the NPs group was significantly lower than that in the Taxotere group $\left(1.25 \mathrm{E}+5\right.$ versus $4.70 \mathrm{E}+5 \mathrm{p} / \mathrm{s} / \mathrm{cm}^{2} / \mathrm{sr}, \mathrm{p}<$ $0.05)$.

Representative bioluminescence images for individual mouse are shown in Figure 5C-G. For the majority of mice, luminescence signals were detected in a small region limited to left lung on day 0 after tumor implantation. On day 5 when treatment was started, tumor cells had spread to a larger area. On day 20, mice in the untreated group had developed very strong luminescence signals, while mice in the Taxotere and NPs groups had significantly lower luminescence levels, with the NP group exhibiting the lowest. The last treatment was administered on day 40 in accordance with the clinical treatment regimen of six weekly i.v. injections. Both the NPs and Taxotere groups showed baseline luminescence intensity levels indicating very little tumor burden. With no further treatment, the Taxotere-treated group was progression free until $\sim$ day 70 , after which tumors relapsed as indicated by an increase in the luminescence intensity. In contrast, tumors in the NP-treated group relapsed only until after day 105. Expression levels of MRP1, LRP, Pgp, GST- $\pi$ of relapsed tumors in the two treatment groups were quantified and compared to that of the original tumors in the untreated group. Expression levels of MRP1, LRP and GST- $\pi$ in relapsed tumors were all significantly higher than that in untreated tumors (supplementary Figure S2), which could indicate acquired drug resistance after treatment.

As shown in Figure 5B, the control group had a median survival of 41 days, which was significantly improved by Taxotere treatment to 131 days. Survival by treatment with the Br-C16-DX NPs was further enhanced to a median survival of 158 days. The improvements were statistically significant compared to the control group ( $p<0.05$ in Log-rank Test).

Nanomedicine. Author manuscript; available in PMC 2015 October 01. 
Improved progression free survival and median survival in the NPs group suggest prolonged systemic circulation and increased tumor accumulation of the Br-C16-DX NPs, as demonstrated in previous pharmacokinetic studies. ${ }^{18}$ For both treatment groups, $55.6 \%$ of the mice had been sacrificed at 158 days after tumor implantation. At this point, the study was terminated as the remaining mice in each group had baseline levels of bioluminescence intensity. Lungs from these surviving mice were harvested and fixed in formalin. Gross images and H\&E staining showed smooth surface and mostly normal lung histology with organized alveoli (Figure 6). Metastasis was shown by positive luminescence in distant tissues only in the control group with a rate of $11.1 \%$ (supplementary Figure S3).

\section{Discussion}

In these current studies, a clinically-relevant orthotopic NSCLC model was established in nude mice. This model was shown to be feasible and reproducible using the dorsal side injection technique, with $94 \%$ lung tumor development rate and $0 \%$ surgery mortality. The Br-C16-DX NPs previously developed in our laboratory were investigated in this model and shown to have superior therapeutic efficacy over commercial Taxotere.

At early stage of model development, we investigated an intrabronchial injection protocol with a $1.2 \mathrm{~cm}$ needle reported by McLemore et al. ${ }^{4}$ After nasal luminescence was observed following injection, the original method was refined by utilizing a one inch blunted needle that was inserted deeper into trachea to allow the tumor cells to reach the lung instead of resulting in nasal deposition due to normal breathing patterns of the mouse. However, this method had surgery mortality of $13 \%$ during intrabronchial injection $(n=15)$. Furthermore, with A549-luc-c8 cells implanted in the lung as confirmed by bioluminescence imaging, lung tumor was successfully developed in only $47 \%$ of the mice three weeks post-injection. Either luminescence signal in trachea or no luminescence was observed in $40 \%$ of the mice. Tumor growth in upper airway is difficult to avoid with intrabronchial injection. Zou et al. observed tumors in bronchus and neck despite careful withdrawal of the needle, and bathing the wound in $70 \%$ ethanol after injection. ${ }^{5}$ Lung tumor was developed in only two out of five mice in their studies.

Intrabronchial injection requires high level surgical skills and specialized devices such as a surgical board and modified needles, which lead to lack of reproducibility of the injection technique and compromised lung tumor development rate. ${ }^{23,}{ }^{24}$ Mice needed to be maintained in a reverse Trendelenburg maneuver using the McLemore protocol to keep tumor cells in the distal airways of the right lung, which requires the use of surgical board and full restraint of all limbs. Vertrees et al. held the mice in a head-up position, and utilized a homemade endotracheal tube, a homemade laryngoscope, together with a piece of PE 50 tubing for cell injection. ${ }^{23}$ Kang et al. reported intrabronchial injection with a needle bent to $135^{\circ}$ angle. The lung tumor development rate was only $20 \%$ even with A549 cells taken from in vivo subcutaneous passaging in nude mice. ${ }^{24}$ However, the simultaneous administration of EDTA improved the lung tumor development rate to $80 \%$ in their studies, presumably due to slight disruption of the lung parenchymal epithelium and surfactant layer. 
Due to surgery complexity and unsatisfactory lung tumor growth, we later investigated tumor injection directly through left chest wall. With an incision on the left chest, cell suspensions were injected directly into the left lung parenchyma $(n=25)$. Subsequent whole-body bioluminescence imaging revealed abdominal luminescence in $20 \%$ of the mice likely due to heart puncture during injection (supplementary Figure S4). For some mice with luminescence only in the lungs, positive luminescence was observed in the heart when organs were imaged following autopsy. Lung tumor development rate by this direct chest injection method was $72 \%$.

To overcome above limitations, the surgical technique was modified wherein the cells were injected from dorsal side over the left lung. Using this modification, the heart was better protected from injection contamination since the injection site was further away (supplementary Figure S4). Abdominal luminescence was observed occasionally but at much lower rate. In a 65-mice study using this dorsal injection technique, abdominal luminescence was observed in only two mice (3\%). Chest cavities were imaged following injection, and were proved to be clean (data not shown). Lung tumor development rate was higher than $90 \%$ and surgery mortality was $0 \%$ in all studies with dorsal side injection. Furthermore, model establishment was easily feasible and very reproducible. Mice could be simply laid on their abdomen during injection with no need to restrain mice or to position them with any special devices or equipment.

An "increase - decrease or plateau - continuous increase" pattern for in vivo bioluminescence was observed repeatedly in the developed model with A549-luc-c8 cells. Similar trend has been observed with intravenous injection of the same cell line into the lungs of SCID mice. ${ }^{25}$ The decrease or plateau phase could be partially attributable to the tissue attenuation of bioluminescence signal rising from the deep tissue, resulting in unproportional increase of signal with the tumor growth. As shown in Figure 3 (lower panel), day 7 and day 14 had increasing PET SUV but similar bioluminescence signal likely due to tissue attenuation in BLI on day 14. It is notable that although there was a slight deviation at lower signal range in the correlation between BLI and PET, a strong linear correlation was observed overall $\left(R^{2}=0.86\right)$. Another potential cause of the plateau phase is a necrotic formation typically in the center of the tumor due to insufficient nutrition supply. In a study with subcutaneous A549 tumor, Ahmed et al. observed an increase in tumor volume followed by a plateau around day 22 to day 25 , and then continuous increase afterwards. ${ }^{26}$ Since tumor volume is measured by geometrical dimensions in subcutaneous model where tissue attenuation is not a concern, this plateau phase could be mainly caused by the formation of a necrotic center in the tumor.

Orthotopic lung tumor growth and lymph node metastasis were shown by increased FDG uptake in PET imaging. The mean SUV of the lung lesion reported here is slightly lower compared to that in subcutaneous A549 model. ${ }^{27-29}$ This is mainly due to the different approach in defining regions of interest in mean SUV measurement. ROI in subcutaneous model only includes tumor tissue, while in our study, due to multiple tumor nodules and small volume in earlier stage, the ROI was defined as the whole lung including tumor tissue and healthy lung tissue, resulting in lower SUV in average. On the other hand, different growth environment in orthotopic model could potentially play a role and lead to the 
different tumor metabolism level compared to the subcutaneous counterpart. Compared to the results from the subcutaneous tumor model, the FDG PET imaging in the orthotopic NSCLC model revealed more heterogeneity of FDG uptake, with SUV ranging from 0.5 to 7.3 , and average SUV (max) of 4.07+/- 1.2. The PET imaging results in the orthotopic model agree with the imaging pattern of human NSCLC with large heterogeneity and high SUV (max) reported in the clinic. ${ }^{30}$

The in vivo efficacy study showed that Br-C16-DX NPs provided a greater therapeutic benefit than Taxotere in the A549-luc-c8 orthotopic lung tumor model. The NP-treated group exhibited delayed tumor relapse from 70 to 105 days, and prolonged median survival from 131 to 158 days as compared to the Taxotere group. These results suggest prolonged systemic circulation and increased tumor accumulation of the Br-C16-DX NPs as was previously established with this $\mathrm{Br}-\mathrm{C} 16-\mathrm{DX} \mathrm{NP}$ formulation in a subcutaneous breast cancer model. ${ }^{18}$ For intravenously administered Taxotere or Br-C16-DX NPs at the same dose, the plasma $\mathrm{AUC}_{0-\infty}$ of NP-formulated $\mathrm{Br}-\mathrm{C} 16-\mathrm{DX}$ was 100 -fold higher than that of Taxotere, and terminal half-life 8.7-fold higher. The DX AUC hydrolyzed from Br-C16-DX was improved by 4.3 -fold compared to Taxotere, and mean resonance time improved by 6.4fold. Furthermore, PK/biodistribution studies of BTM NPs with a drug conjugate were performed in the developed orthotopic NSCLC model. The plasma half-life of the drug conjugate from BTM NPs was prolonged to 7.9 hour compared to free drug at 0.8 hour, producing a significantly higher dose-normalized $\mathrm{AUC}_{0-96 \mathrm{~h}}$ of $20405.5(\mathrm{~h} * \mathrm{ng} / \mathrm{ml}) /(\mathrm{mg} / \mathrm{kg})$ for the NP-delivered conjugate compared to $878.0(\mathrm{~h} * \mathrm{ng} / \mathrm{ml}) /(\mathrm{mg} / \mathrm{kg})$ for the free drug. In addition, the half-life of the drug conjugate in tumor-bearing lungs was 2.7-fold longer than free drug (80.9 versus 30.3 hour) and the dose-normalized $\mathrm{AUC}_{0-96 \mathrm{~h}}$ was 12.6-fold greater (34689.4 versus $2762.0(\mathrm{~h} * \mathrm{ng} / \mathrm{ml}) /(\mathrm{mg} / \mathrm{kg})$ ). These results demonstrated that BTM NPs significantly prolonged circulation time and increased drug exposure of the delivered drug to the target tissue.

The linear correlation between BLI and PET (Figure 3, $\mathrm{R}^{2}=0.86$ ) in the orthotopic NSCLC model supported the use of BLI to monitor tumor growth and to compare different treatment groups and their response. BLI is also more time- and cost-effective than PET. In the efficacy study, bioluminescence from A549-luc-c8 provided a reliable signal for tumor growth up to more than 150 days. In the untreated group, both primary tumor growth and late stage metastasis to lymph nodes were identified by positive bioluminescence signal. In both treatment groups, suppression of original tumors, progression-free period, and tumor relapse were shown by a decrease in luminescence to nearly baseline levels followed by an increase in the luminescence upon tumor regrowth. Further, histological examination after autopsy supported the imaging results.

Expression levels of drug resistance-related proteins were significantly higher in relapsed tumors compared to untreated tumors. Nanomedicines have the potential to overcome drug resistance through inhibition of drug efflux transporters, receptor-mediated endocytosis, or unclear mechanisms. ${ }^{31-33}$ Specifically, BTM NPs have been previously proven to inhibit Pgp and deplete ATP. ${ }^{34} \mathrm{Br}-\mathrm{C} 16-\mathrm{DX}$ NPs to treat resistant lung tumor could be further investigated. 


\section{Supplementary Material}

Refer to Web version on PubMed Central for supplementary material.

\section{Acknowledgments}

This work was supported by NIH-NCI R01 CA115197 and NIH-NCI U54 CA151652 to RJM. The BRIC imaging facility is supported in part by NCI grant P30-CA016086-35-37, and U54-CA151652-01-03. Ms. Peng is the recipient of Translational Medicine training fellowship at UNC-Chapel Hill supported by "Med into Grad" training grant from the Howard Hughes Medical Institute.

The authors are very grateful to the Animal Studies Core at UNC-Chapel Hill, especially Charlene M. Santos, for assistance with animal studies. The authors would also like to acknowledge Animal Histopathology Core at Lineberger Comprehensive Cancer Center, especially Yongjuan Xia, for histology staining. The authors would like to thank Microscopy Services Laboratory at UNC-Chapel Hill for assistance with microscopic observations. Thanks to Kevin Guley for imaging support, and BRIC (Biomedical Research Imaging Center) Small Animal Imaging facility for imaging service.

\section{References}

1. Siegel R, Naishadham D, Jemal A. Cancer statistics, 2012. CA Cancer J Clin. 2012; 62:10-29. [PubMed: 22237781]

2. Kuo TH. Site-specific chemosensitivity of human small-cell lung carcinoma growing orthotopically compared to subcutaneously in SCID mice: the importance of orthotopic models to obtain relevant drug evaluation data. Anticancer Res. 1993; 13:627-30. [PubMed: 8391244]

3. Wilmanns C. Orthotopic and ectopic organ environments differentially influence the sensitivity of murine colon carcinoma cells to doxorubicin and 5-fluorouracil. Int J Cancer. 1992; 52:98-104. [PubMed: 1500231]

4. McLemore TL, Liu MC, Blacker PC, Gregg M, Alley MC, Abbott BJ, et al. Novel Intrapulmonary Model for Orthotopic Propagation of Human Lung Cancers in Athymic Nude Mice. Cancer Res. 1987; 47:5132-40. [PubMed: 3621199]

5. Zou Y, Fu H, Ghosh S, Farquhar D, Klostergaard J. Antitumor Activity of Hydrophilic Paclitaxel Copolymer Prodrug Using Locoregional Delivery in Human Orthotopic Non Small Cell Lung Cancer Xenograft Models. Clin Cancer Res. 2004; 10:7382-91. [PubMed: 15534115]

6. Hasenpusch G, Pfeifer C, Aneja MK, Wagner K, Reinhardt D, Gilon M, et al. Aerosolized BC-819 Inhibits Primary but Not Secondary Lung Cancer Growth. PLoS One. 2011; 6:e20760. [PubMed: 21687669]

7. Onn A, Isobe T, Itasaka S, Wu W, O'Reilly MS, Ki Hong W, et al. Development of an Orthotopic Model to Study the Biology and Therapy of Primary Human Lung Cancer in Nude Mice. Clin Cancer Res. 2003; 9:5532-9. [PubMed: 14654533]

8. Podesta JE, Al-Jamal KT, Herrero MA, Tian B, Ali-Boucetta H, Hegde V, et al. Antitumor Activity and Prolonged Survival by Carbon-Nanotube-Mediated Therapeutic siRNA Silencing in a Human Lung Xenograft Model. Small. 2009; 5:1176-85. [PubMed: 19306454]

9. Azzoli CG, Baker S, Temin S, Pao W, Aliff T, Brahmer J, et al. American Society of Clinical Oncology Clinical Practice Guideline Update on Chemotherapy for Stage IV Non-Small-Cell Lung Cancer. J Clin Oncol. 2009; 36:6251-66. [PubMed: 19917871]

10. Ramanlal Chaudhari K, Kumar A, Megraj Khandelwal VK, Ukawala M, Manjappa AS, Mishra AK, et al. Bone metastasis targeting: A novel approach to reach bone using Zoledronate anchored PLGA nanoparticle as carrier system loaded with Docetaxel. J Controlled Release. 2012; 158:470_ 8.

11. Naik S, Patel D, Chuttani K, Mishra AK, Misra A. In vitro mechanistic study of cell death and in vivo performance evaluation of RGD grafted PEGylated docetaxel liposomes in breast cancer. Nanomedicine. 2012; 8:951-62. [PubMed: 22115600]

12. Venishetty VK, Komuravelli R, Kuncha M, Sistla R, Diwan PV. Increased brain uptake of docetaxel and ketoconazole loaded folate-grafted solid lipid nanoparticles. Nanomedicine. 2013; 9:111-21. [PubMed: 22426195] 
13. Ungaro F, Conte C, Ostacolo L, Maglio G, Barbieri A, Arra C, et al. Core-shell biodegradable nanoassemblies for the passive targeting of docetaxel: features, antiproliferative activity and in vivo toxicity. Nanomedicine. 2012; 8:637-46. [PubMed: 21889924]

14. Feng L, Wu H, Ma P, Mumper RJ, Benhabbour SR. Development and optimization of oil-filled lipid nanoparticles containing docetaxel conjugates designed to control the drug release rate in vitro and in vivo. Int J Nanomedicine. 2011; 6:2545-56. [PubMed: 22072889]

15. Ali S, Ahmad I, Peters A, Masters G, Minchey S, Janoff A, et al. Hydrolyzable hydrophobic taxanes: synthesis and anti-cancer activities. Anti-Cancer Drugs. 2001; 12:117-28. [PubMed: 11261884]

16. Foltz CJ, Ullman-Cullere M. Guidelines for assessing the health and condition of mice. Lab Animal. 1999; 28:28-32.

17. Huynh L, Leroux J-C, Allen C. Enhancement of docetaxel solubility via conjugation of formulation-compatible moieties. Org Biomol Chem. 2009; 7:3437-46. [PubMed: 19675898]

18. Feng L, Benhabbour SR, Mumper RJ. Oil-filled Lipid Nanoparticles Containing 2'-(2bromohexadecanoyl)-docetaxel for the Treatment of Breast Cancer. Adv Healthcare Mater. 201310.1002/adhm.201300017

19. Dong X, Mattingly CA, Tseng M, Cho M, Adams VR, Mumper RJ. Development of new lipidbased paclitaxel nanoparticles using sequential simplex optimization. Eur J Pharm Biopharm. 2009; 72:9-17. [PubMed: 19111929]

20. Ma P, Dong X, Swadley CL, Gupte A, Leggas M, Ledebur HC, et al. Development of idarubicin and doxorubicin solid lipid nanoparticles to overcome Pgp-mediated multiple drug resistance in leukemia. J Biomed Nanotechnol. 2009; 5:151-61. [PubMed: 20055093]

21. Ma P, Benhabbour SR, Feng L, Mumper RJ. 2'-Behenoyl-paclitaxel conjugate containing lipid nanoparticles for the treatment of metastatic breast cancer. Cancer Lett. 2013; 334:253-62. [PubMed: 22902506]

22. Madero-Visbal RA, Colon JF, Hernandez IC, Limaye A, Smith J, Lee CM, et al. Bioluminescence imaging correlates with tumor progression in an orthotopic mouse model of lung cancer. Surg Oncol. 2012; 21:23-9. [PubMed: 20801643]

23. Vertrees RA, Deyo DJ, Quast M, Lightfoot KM, Boor PJ, Zwischenberger JB. Development of a Human to Murine Orthotopic Xenotransplanted Lung Cancer Model. J Invest Surg. 2000; 13:349_ 58. [PubMed: 11202012]

24. Kang Y, Omura M, Suzuki A, Oka T, Nakagami Y, Cheng C, et al. Development of an orthotopic transplantation model in nude mice that simulates the clinical features of human lung cancer. Cancer Sci. 2006; 97:996-1001. [PubMed: 16984373]

25. Wu S, Kasim V, Kano MR, Tanaka S, Ohba S, Miura Y, et al. Transcription Factor YY1 Contributes to Tumor Growth by Stabilizing Hypoxia Factor HIF-1a in a p53-Independent Manner. Cancer Res. 2013; 73:1787-99. [PubMed: 23328582]

26. Ahmed AU, Schmidt RL, Park CH, Reed NR, Hesse SE, Thomas CF, et al. Effect of Disrupting Seven-in-Absentia Homolog 2 Function on Lung Cancer Cell Growth. J Natl Cancer Inst. 2008; 100:1606-29. [PubMed: 19001609]

27. Kim S-L, Kim E-M, Cheong S-J, Lee C-M, Kim DW, Jeong H-J, et al. The effect of PPAR- $\gamma$ agonist on 18F-FDG uptake in tumor and macrophages and tumor cells. Nucl Med Biol. 2009; 36:427-33. [PubMed: 19423011]

28. Ong LC, Jin Y, Song IC, Yu S, Zhang K, Chow PK. 2-[18F]-2-deoxy-D-glucose (FDG) uptake in human tumor cells is related to the expression of GLUT-1 and hexokinase II. Acta Radiol. 2008; 49:1145-53. [PubMed: 18979289]

29. Guo R, Liang S, Ma Y, Shen H, Xu H, Li B. Evaluation of biodistribution and antitumor effects of (188)Re-rhk5 in a mouse model of lung cancer. Oncol Lett. 2011; 2:865-70. [PubMed: 22866142]

30. Machtay M, Duan F, Siegel BA, Snyder BS, Gorelick JJ, Reddin JS, et al. Prediction of Survival by [18F]Fluorodeoxyglucose Positron Emission Tomography in Patients With Locally Advanced Non-Small-Cell Lung Cancer Undergoing Definitive Chemoradiation Therapy: Results of the ACRIN 6668/RTOG 0235 Trial. J Clin Oncol. 2013; 31:3823-30. [PubMed: 24043740]

31. Gu YJ, Cheng JP, Man CWY, Wong WT, Cheng SH. Gold-doxorubicin nanoconjugates for overcoming multidrug resistance. Nanomedicine. 2012; 8:204-11. [PubMed: 21704592] 
32. Yan F, Zhang C, Zheng Y, Mei L, Tang LN, Song CX, et al. The effect of poloxamer 188 on nanoparticle morphology, size, cancer cell uptake, and cytotoxicity. Nanomedicine. 2010; 6:1708. [PubMed: 19447200]

33. Kabanov AV, Batrakova EV, Alakhov VY. Pluronic $®$ block copolymers for overcoming drug resistance in cancer. Adv Drug Delivery Rev. 2002; 54:759-79.

34. Dong X. Doxorubicin and Paclitaxel-Loaded Lipid-Based Nanoparticles Overcome Multidrug Resistance by Inhibiting P-Glycoprotein and Depleting ATP. Cancer Res. 2009; 69:3918-26. [PubMed: 19383919] 
A
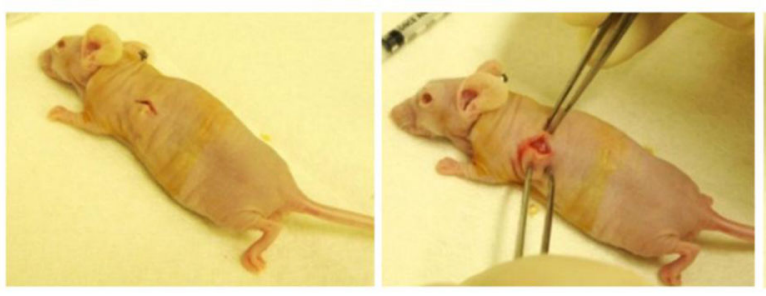

B

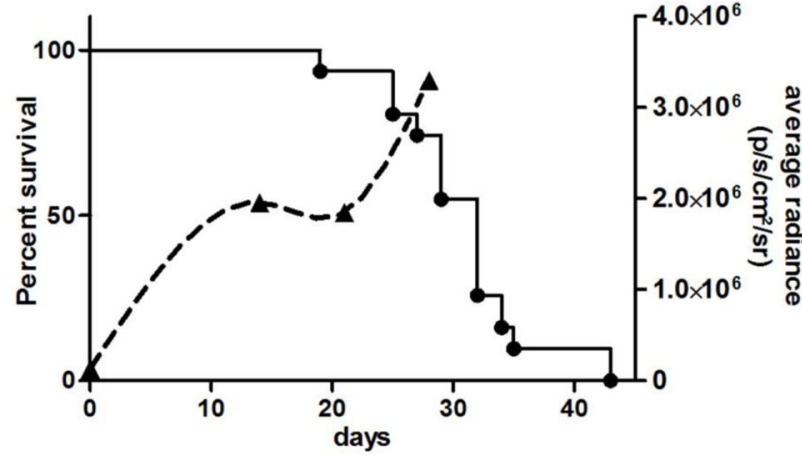

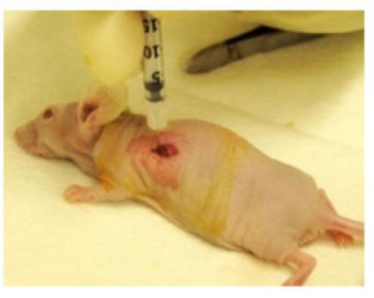

C

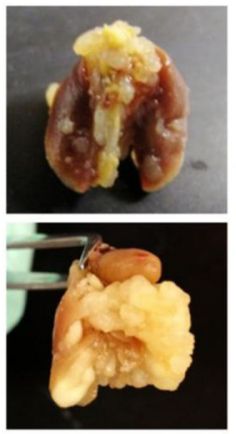

Figure 1.

Orthotopic lung tumor implantation and survival study. (A)Dorsal side lung tumor implantation procedure. (B) Survival curve (left y-axis, circle) and whole-body bioluminescence monitoring (right y-axis, triangle) in survival study (n=34). (C) Gross images of tumor-bearing lungs with disorganized tumor tissue. 

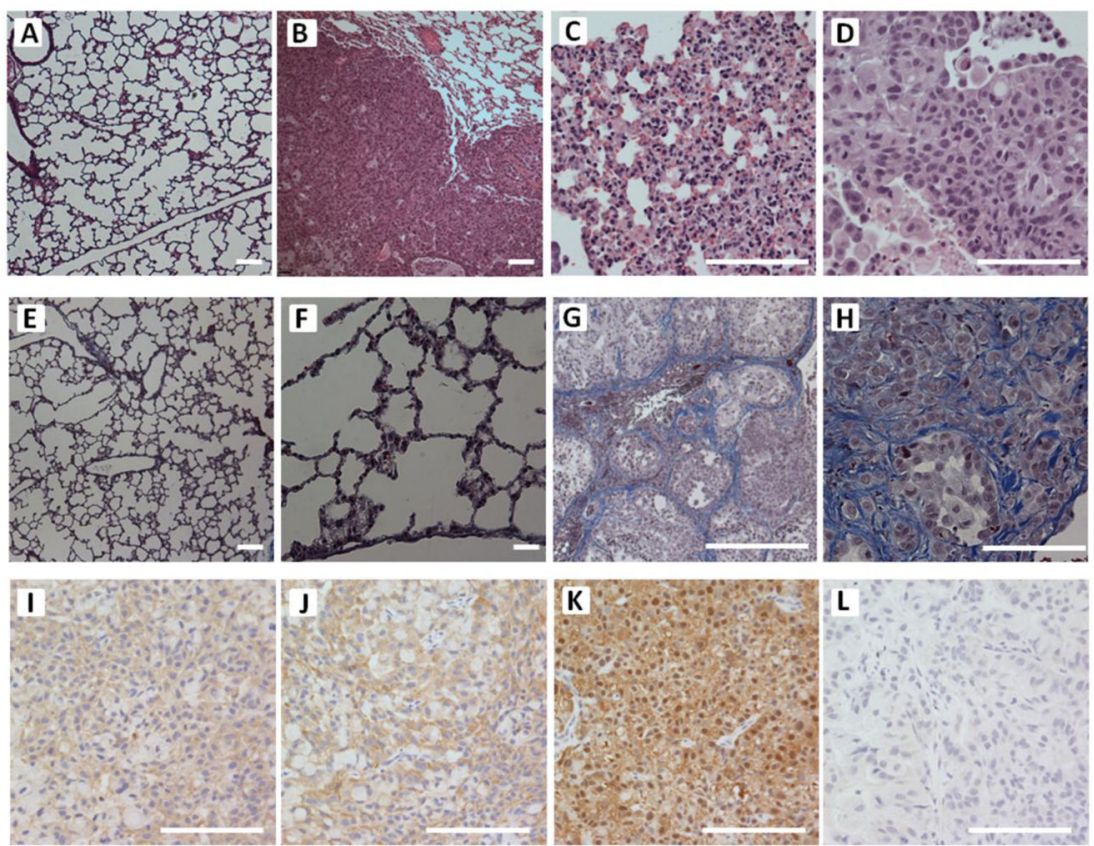

Figure 2.

Histology characterization of orthotopic lung tumors. (A-D) H\&E staining of healthy lung (A) and tumor-bearing lungs (B, C, D). (E-H)Masson's Trichrome Staining of healthy lung (E and $\mathbf{F}$ ) and tumor-bearing lungs ( $\mathbf{G}$ and $\mathbf{H})$. (I-L) Immunohistochemistry for MRP1 (I), LRP (J), GST- $\pi$ (K) and Pgp (L). Scale bar, $120 \mu \mathrm{m}$. 

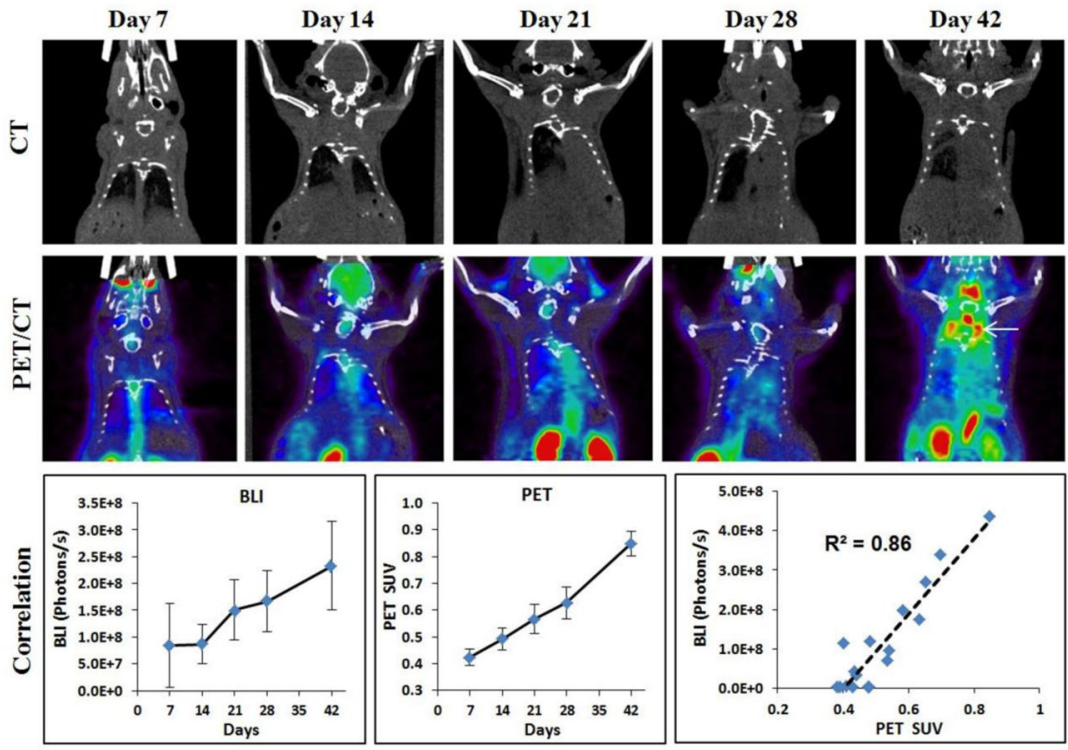

Figure 3.

MicroPET/CT imaging and correlation with optial imaging. Upper panel, CT imaging of the same mouse from day 7 to day 42. Middle panel, PET/CT overlay with an arrow in day 42 image indicating high FDG updake in lymph nodes. Lower panel, quantified signal from bioluminescence imaging (BLI, left), FDG PET imaging (middle), and the correlation of these two imaging approaches (right, $\mathrm{R}^{2}=0.86$ ). 


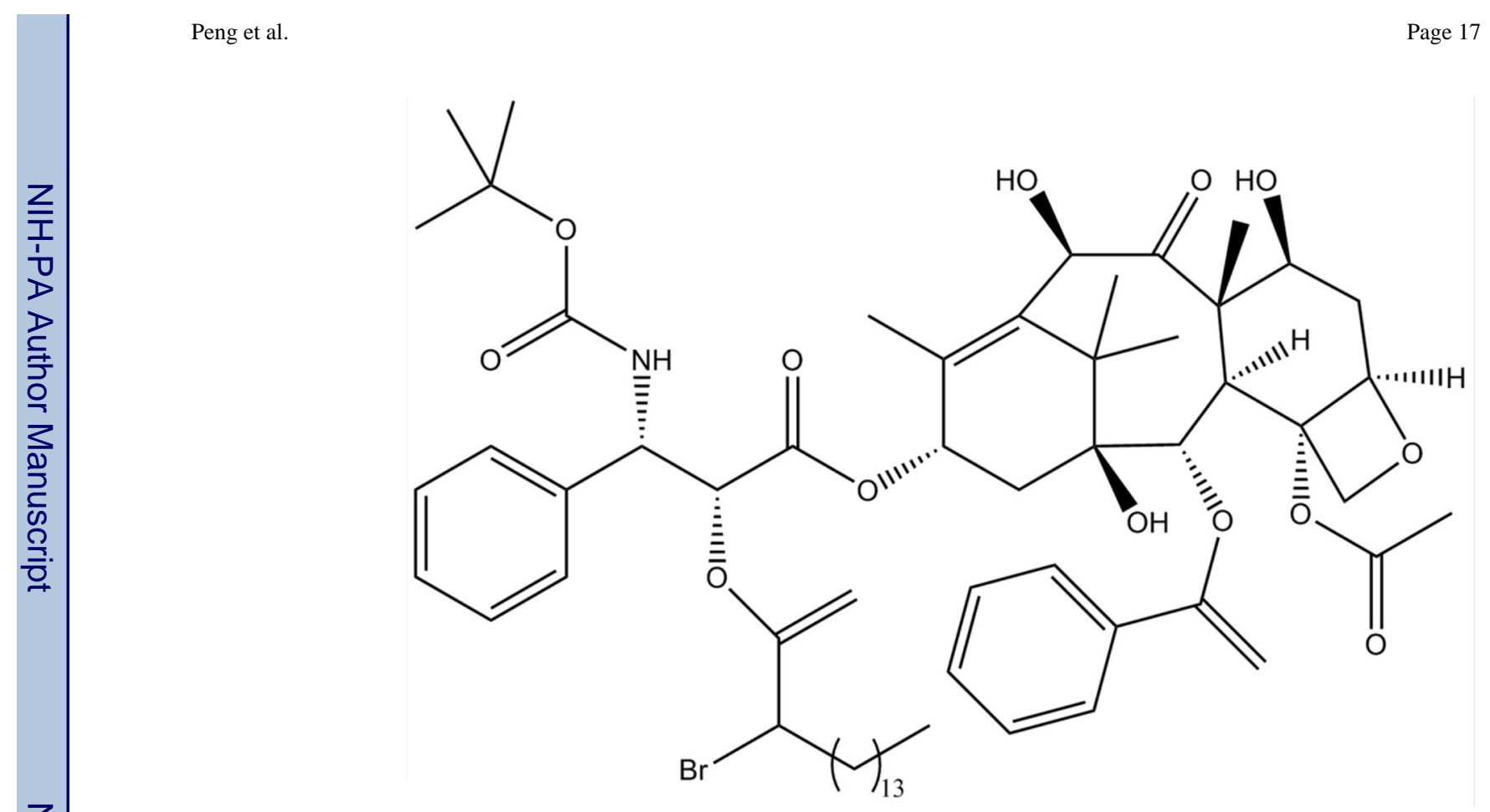

Figure 4.

Structure of Br-C16-DX. 

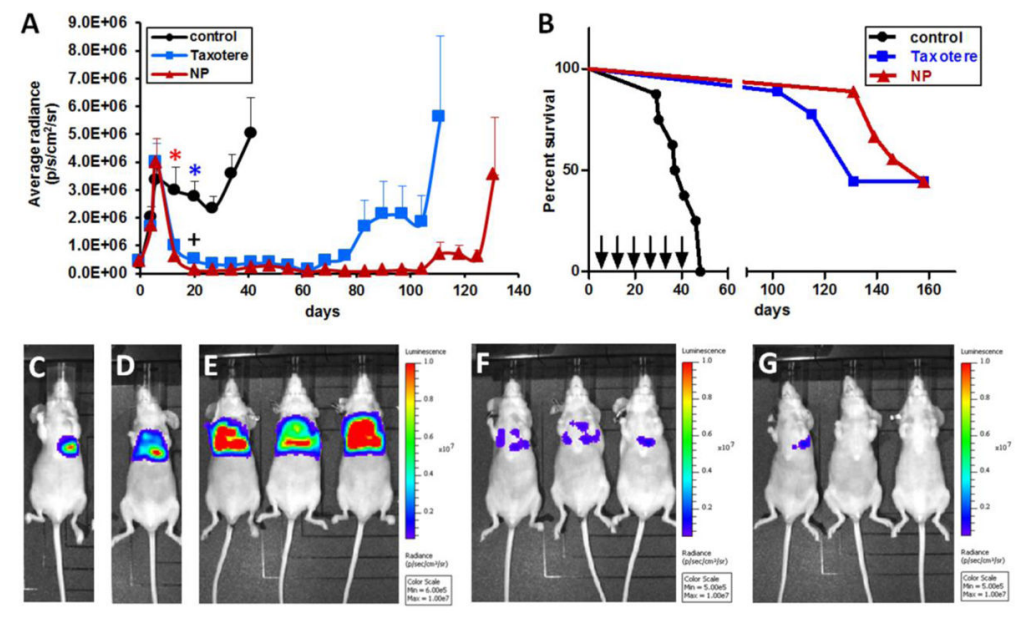

Figure 5.

Efficacy study with Taxotere and Br-C16-DX NPs. (A) Quantified average luminescence levels in different groups. Red asterisk, NPs group vs. control group on day 13, $\mathrm{p}<0.05$. Blue asterisk, Taxotere group versus control group on day $20, \mathrm{p}<0.05$. +, NPs group versus Taxotere group on day 20, $\mathrm{p}<0.05$. (B) Survival curve. Tail vein injections are indicated by the arrows. (C) and (D), Representative whole-body bioluminescence image on day 0 (C) and day 5 (D). (E-G), representative bioluminescence images on day 20 in control (E), Taxotere (F), and NP group (G). 

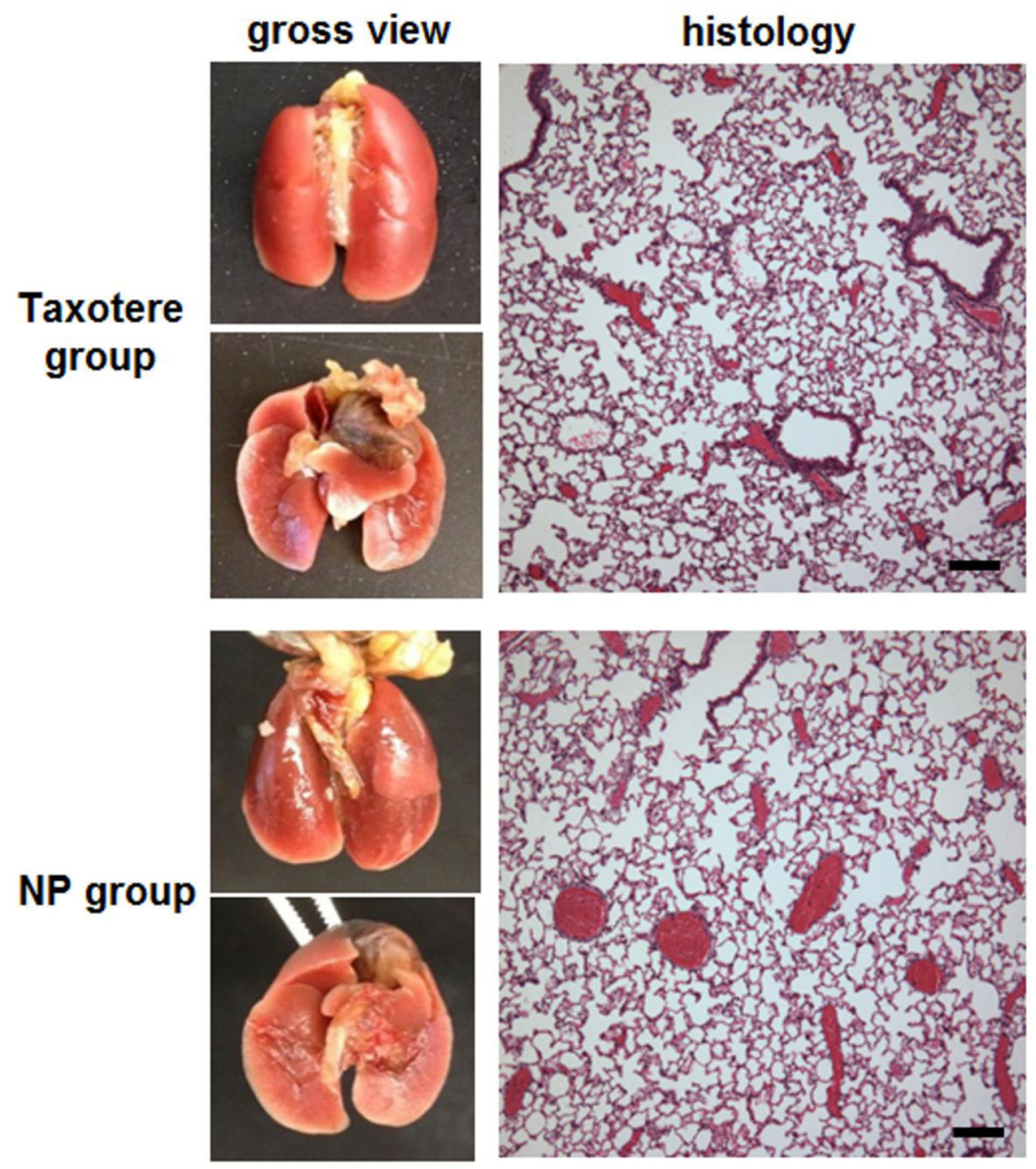

Figure 6.

Gross images and H\&E staining of lungs from surviving mice in two treatment groups. Scale bar, $60 \mu \mathrm{m}$. 